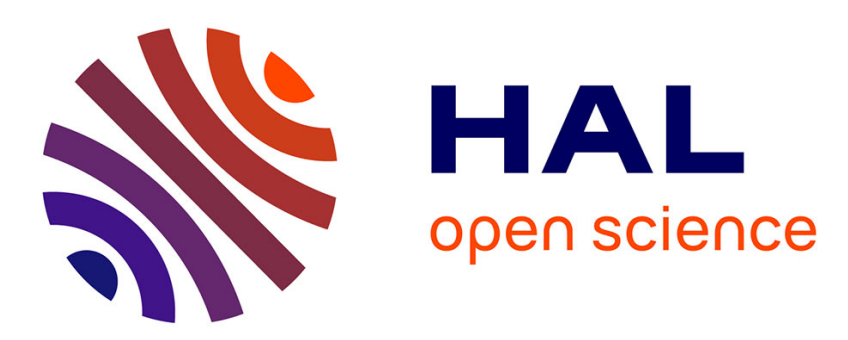

\title{
Hutchinson revisited: Patterns of density regulation and the coexistence of strong competitors
}

\author{
Tamara Münkemüller, Harald Bugmann, Karin Johst
}

\section{To cite this version:}

Tamara Münkemüller, Harald Bugmann, Karin Johst. Hutchinson revisited: Patterns of density regulation and the coexistence of strong competitors. Journal of Theoretical Biology, 2009, 259 (1), pp.109. 10.1016/j.jtbi.2009.03.010 . hal-00554588

\section{HAL Id: hal-00554588 \\ https://hal.science/hal-00554588}

Submitted on 11 Jan 2011

HAL is a multi-disciplinary open access archive for the deposit and dissemination of scientific research documents, whether they are published or not. The documents may come from teaching and research institutions in France or abroad, or from public or private research centers.
L'archive ouverte pluridisciplinaire HAL, est destinée au dépôt et à la diffusion de documents scientifiques de niveau recherche, publiés ou non, émanant des établissements d'enseignement et de recherche français ou étrangers, des laboratoires publics ou privés. 


\section{Author's Accepted Manuscript}

Hutchinson revisited: Patterns of density regulation and the coexistence of strong competitors

Tamara Münkemüller, Harald Bugmann, Karin Johst

PII:

S0022-5193(09)00120-9

DOI: doi:10.1016/j.jtbi.2009.03.010

Reference: YJTBI 5496

To appear in: $\quad$ Journal of Theoretical Biology

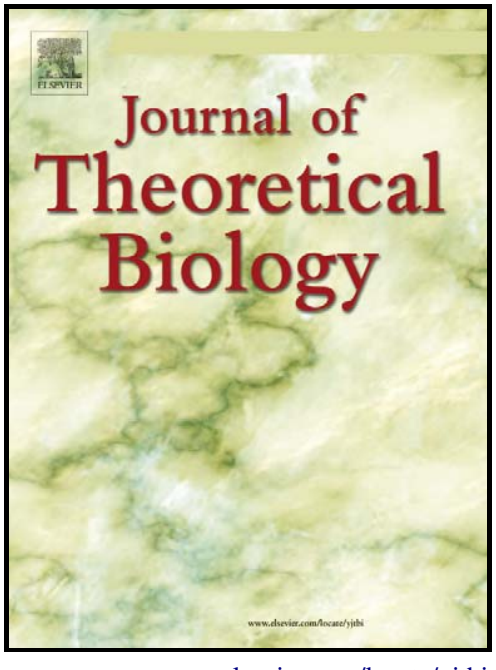

www.elsevier.com/locate/yjtbi

Received date: 24 October 2008

Revised date: 23 February 2009

Accepted date: $\quad 9$ March 2009

Cite this article as: Tamara Münkemüller, Harald Bugmann and Karin Johst, Hutchinson revisited: Patterns of density regulation and the coexistence of strong competitors, Journal of Theoretical Biology (2009), doi:10.1016/j.jtbi.2009.03.010

This is a PDF file of an unedited manuscript that has been accepted for publication. As a service to our customers we are providing this early version of the manuscript. The manuscript will undergo copyediting, typesetting, and review of the resulting galley proof before it is published in its final citable form. Please note that during the production process errors may be discovered which could affect the content, and all legal disclaimers that apply to the journal pertain. 


\section{Hutchinson revisited: Patterns of density regulation and the}

\section{2 coexistence of strong competitors}

3

4 Tamara Münkemüller ${ }^{\mathrm{a}, \mathrm{b}^{*}}$, Harald Bugmann $^{\mathrm{b}}$ \& Karin Johst $^{\mathrm{a}}$

5

6

$7{ }^{\text {a }}$ Helmholtz Centre for Environmental Research - UFZ, Department of Ecological

8 Modelling, Permoserstr. 15, 04301 Leipzig, Germany, tamara.muenkemueller@ufz.de,

9 karin.johst@ufz.de

$10{ }^{\mathrm{b}}$ Forest Ecology, Department of Environmental Sciences, ETH Zurich, CH-8092

11 Zurich, Switzerland, harald.bugmann@env.ethz.ch

12

$13{ }^{*}$ Corresponding author:

14 Tamara Münkemüller, telephone: +49 (0) 341 2351707, Fax: +49 (0) 3412351473 


\section{Abstract}

2 Ecologists have long been searching for mechanisms of species coexistence, particularly

3 since G.E. Hutchinson raised the 'paradox of the plankton'. A promising approach to

4 solve this paradox and to explain the coexistence of many species with strong niche

5 overlap is to consider over-compensatory density regulation with its ability to generate

6 endogenous population fluctuations.

7 Previous work has analyzed the role of over-compensation in coexistence based

8 on analytical approaches. Using a spatially explicit time-discrete simulation model, we

9 systematically explore the dynamics and conditions for coexistence of two species. We

10 go beyond the analytically accessible range of models by studying the whole range of

11 density regulation from under- to very strong over-compensation and consider the

12 impact of spatial structure and temporal disturbances. In particular, we investigate how

13 coexistence can emerge in different types of population growth models.

14 We show that two strong competitors are able to coexist if at least one species

15 exhibits over-compensation. Analyzing the time series of population dynamics reveals

16 how the differential responses to density fluctuations of the two competitors lead to

17 coexistence: The over-compensator generates density fluctuations but is the inferior

18 competitor at strong amplitudes of those fluctuations; the competitor therefore becomes

19 frequent and dampens the over-compensator's amplitudes, but it becomes inferior under

20 dampened fluctuations.

21 These species interactions cause a dynamic alternation of community states with

22 long-term persistence of both species. We show that a variety of population growth

23 models is able to reproduce this coexistence although the particular parameter ranges

24 differ among the models. Spatial structure influences the probability of coexistence but 
1 coexistence is maintained for a broad range of dispersal parameters.

The flexibility and robustness of coexistence through over-compensation

3 emphasize the importance of non-linear density dependence for species interactions, and

4 they also highlight the potential of applying more flexible models than the classical

$5 \quad$ Lotka-Volterra equations in community ecology.

6

7 Keywords: Over-compensation, under-compensation, relative nonlinearity, Lotka-

8 Volterra, complex dynamics 


\section{1. Introduction}

2 The search for mechanisms of species coexistence has a long tradition in ecology.

3 Already Lotka (1925) and Volterra (1926) investigated this question with a theoretical

4 competition model in the early $20^{\text {th }}$ century and found that intraspecific competition

5 must be greater than interspecific competition to facilitate coexistence of two species.

6 Gause (1934) complemented this finding by experimental work on Paramecium species

7 and concluded that two species competing for the same resources cannot stably coexist.

8 Hutchinson answered by raising the 'paradox of the plankton' (Hutchinson 1961). He

9 pointed to the apparent contradiction between the principle of 'competitive exclusion'

10 (Gause 1934) and the existence of many highly diverse natural communities living on

11 strongly limited numbers of resources. This contradiction brings up the question which

12 mechanisms enable the number of coexisting species to exceed the number of available

13 resources (Armstrong and McGehee 1976; Lundberg et al. 2000; Szabo and Meszena

14 2006).

15 Coexistence mechanisms can be classified into equalizing and stabilizing 16 mechanisms (Chesson 2000). Equalizing mechanisms build on minimizing differences

17 in average fitness while stabilizing mechanisms rely on increased intra- compared to 18 interspecific competition strength, which disproportionately reduces the average fitness

19 of the more abundant species (Chesson 1994; Chesson 2000). Among the stabilizing

20 coexistence mechanisms, some depend on heterogeneous distributions of species in

21 space (i.e. spatial storage effects, Shmida and Ellner 1984; Hanski and Woiwod 1993;

22 Chesson 2000; Neuhauser and Pacala 1999). Others depend on fluctuations of

23 population densities in time and can foster coexistence via (1) temporal storage effects

24 or (2) different nonlinear responses to common fluctuating abiotic or biotic limiting 
1 factors (Chesson 1994; Chesson 2003). In this context, the term 'biotic' refers to

2 explicitly modelled resource dynamics while the term 'abiotic' refers to a constant

3 limiting factor.

4 Temporal storage effects are based on external variations of the environment

5 causing species' density fluctuations, e.g. through seasonal variations in resource

6 growth or strong and frequent disturbances (Smith 1981; Grover 1990; Anderies and

7 Beisner 2000; Abrams 2004).

8 Fluctuations of abiotic resources can emerge from different nonlinear consumer

9 responses and lead to the coexistence of many species on a handful of resources in

10 continuous-time models (Huisman and Weissing 1999). At least three resources are

11 needed, and there is an ongoing debate regarding the parameter space under which this

12 behaviour can be observed (Armstrong and McGehee 1976; Armstrong and McGehee

13 1980; Schippers et al. 2001; Huisman et al. 2001). In contrast, competition for biotic

14 resources can lead to the coexistence of two or more consumers on a single resource

15 (Armstrong and McGehee 1980; Zicarelli 1975; Abrams 2004; Kaitala et al. 1999). In

16 this case, coexistence requires at least two differences in species traits: first in the type

17 of functional response in resource use, and second in the minimum resource

18 requirements.

19 It is well known that fluctuations in population densities can also occur through

20 over-compensatory density regulation (May 1975; May 1976; Johst et al. 2008). In this

21 case, individuals scramble for coveted resources and populations over-compensate

22 deviations from carrying capacities which results in frequent peaks and crashes in

23 abundances (cyclic and chaotic dynamics). With compensatory density regulation,

24 individuals follow strategies that effectively avoid resource over-use and population 
1 sizes fluctuate around the carrying capacity only due to stochastic events (equilibrium

2 dynamics). With under-compensatory density regulation, populations under-compensate

3 deviations from carrying capacities and the adjustment of population sizes to carrying

4 capacities is slow (delayed dynamics). For example, storage capacities may lead to a

5 delayed response if resources decline.

6 Over-compensatory density regulation and the resulting non-linear dynamics and

7 fluctuations have been investigated extensively with respect to single species

8 persistence (e.g., Ripa and Lundberg 2000; Murrell et al. 2002; Münkemüller and Johst

9 2006; Münkemüller and Johst 2007). However, few studies explored their contribution

10 to species coexistence. Damgaard (2004) expanded the Lotka-Volterra model of

11 coexistence (Volterra 1926; Lotka 1925) to a situation where both species exhibited the

12 same over-compensatory density regulation and showed that - in agreement with the

13 classical Lotka-Volterra competition model - coexistence was dependent on stronger

14 intraspecific than interspecific competition (i.e. only partial niche overlap). Other

15 authors found that differences in the types of density regulation can facilitate

16 coexistence under certain assumptions of landscape configuration and interaction

17 behaviour (Getz 1996; Johansson and Sumpter 2003; Edmunds et al. 2003; Kuang and

18 Chesson 2008). Analytically analyzing coexistence conditions at the limit of periodic

19 behaviour, Adler (1990) and Cushing (2007) provided evidence of oscillatory

20 coexistence of two species with complete niche overlap, i.e. intraspecific competition

21 equalling interspecific competition.

22 However, to date no study has systematically explored the robustness of

23 coexistence through over-compensation for a wide variety of density regulation types

24 (beyond the cyclic range including chaotic dynamics) and in a context that examines the 
1 role of temporal disturbances and spatial habitat fragmentation. Here, we investigate

2 how different population growth models are able to display this coexistence.

3 We conduct these investigations using a time-discrete simulation model of

4 population growth. In a first step, we study community dynamics and analyse the

5 species-specific time series to better understand when and how two strong competitors

6 are able to coexist through over-compensatory density regulation. In a second step, we

7 investigate the impact of demographic parameters such as growth rate and carrying

8 capacity in four different population growth models. Finally, we investigate whether

9 space matters and how species' dispersal abilities (emigration rate and dispersal

10 mortality) influence coexistence through over-compensation. 


\section{2. Model description}

2 We simulate a two-species community with time-discrete dynamics. The species can

3 differ in their density regulation type, but all other attributes are identical. Niches of the

4 species can overlap completely, only partly, or not at all. The species live either in a

5 homogeneous landscape or in a fragmented landscape with four patches connected via

6 dispersal. an extended version of the Maynard Smith and Slatkin equation (MSS model) unless noted otherwise (1973; see also Hassell and Comins 1976):

10

$$
N_{A}(t+1) \sim \operatorname{Pois}\left(\frac{N_{A}(t) \cdot R}{\left(1+(R-1)\left(\left(N_{A}(t)+a N_{B}(t)\right) / K\right)^{b_{A}}\right)}\right)
$$

11

$$
N_{B}(t+1) \sim \operatorname{Pois}\left(\frac{N_{B}(t) \cdot R}{\left(1+(R-1)\left(\left(N_{B}(t)+a N_{A}(t)\right) / K\right)^{b_{B}}\right)}\right)
$$

13 The population sizes of species $A$ and $B$ at time $t$ are given by $N_{A}(t)$ and $N_{B}(t)$. Both species have the same maximum growth rate, $R$, and common local carrying capacity $K$.

15 The competition coefficient $a$ describes the strength of interspecific relative to 16 intraspecific competition, to which we refer using the term 'niche overlap'. It can be 17 derived from the overlap of resource utilization curves along a resource axis (cf.

18 Abrams et al. 2008). For most simulations we keep inter- and intraspecific competition 19 strength equal (competition coefficient $a=1$ ). Exceptions are the analyses without 
1 interspecific competition ( $a=0$; Fig. 2) and those where we explore the effect of the 2 competition coefficient on coexistence (systematic variation of $a$; cf. Fig. 5). Both

3 species differ only in their type of density regulation, which is characterized by $b_{A}$ and

$4 b_{B}$ ( $b<1$ corresponds to under-compensating, $b=1$ to compensating, and $b>1$ to over-

5 compensating density regulation). In this way, a wide range of combinations of density

6 regulation types from under-compensation to strong over-compensation can be studied

7 at given growth rates, carrying capacities and competition coefficients. We account for

8 demographic stochasticity by using random numbers from a Poisson distribution. We

9 independently and randomly initialize populations for each species by drawing from a

10 uniform distribution between 10 and $10+K$ individuals.

11 For the comparative analysis we implemented further growth models. To

12 simplify matters, we write them in a similar way with $N_{\text {all }}$ referring to the weighted sum

13 of individuals of both species (with weighting factor $a$, see MSS model for

14 comparison): Similar to the MSS model, dynamics can be varied from equilibrium to

15 cyclic and chaotic dynamics independently of $R$ simply by increasing the value of

16 parameter $b$ (viz., type of density regulation).

17 Hassell model:

$$
N(t+1)=\frac{R \cdot N(t)}{\left(1+\left(R^{1 / b}-1\right) N_{\text {all }}(t) / K\right)^{b}}
$$

18 Generalized Ricker model: $\quad N(t+1)=N(t) \cdot e^{\ln R \cdot\left(1-\left(\frac{N_{\text {all }}}{K}\right)^{b}\right)}$

19 Gompertz type model: $\quad N(t+1)=N(t) \cdot e^{\ln R \cdot\left(1-\left(\frac{\ln N_{\text {all }}}{\ln K}\right)^{b}\right)}$

In the fragmented landscape with four patches, dispersal occurs after local

21 population growth. The population size after dispersal $N_{i}(t, d=1)$ is equal to the one prior

22 to dispersal, $N_{i}(t, d=0)$, minus the number of emigrants, $N_{e m i, i}(t)$, and plus the number of 
1 immigrants. The number of emigrants per patch is drawn from a binomial distribution

2 with $N_{i}(t, d=0)$ number of trials and emigration rate $p_{\text {emi }}$. The number of immigrants

3 from patch $i$ to patch $j$ is drawn from a multinomial distribution with $N_{e m i, i}(t)$ trials and

4 transfer probability $p_{i j}$. The matrix of transfer probabilities, with entries $p_{i j}$, describes the

5 probability to move from patch $i$ to patch $j$. Values decrease exponentially with the

6 Euclidean distance between patches, $D_{i j}$, measured in units of grid cells:

7

$8 \quad p_{i j}=\frac{\exp \left(-\frac{1}{D D} D_{i j}\right)}{\sum_{j} \exp \left(-\frac{1}{D D} D_{i j}\right)} \cdot \exp \left(-m D_{i j}\right)$

10 The mean distance over which both species are able to disperse is defined by $D D$, and

11 the dispersal mortality rates are defined by $m$. The denominator scales the transfer

12 probabilities $p_{i j}$ such that they add up to one over all $j$ in the absence of dispersal

13 mortality (i.e. for the special case $m=0$ ). Emigration probability, dispersal distance and

14 dispersal mortality rate $m$ are equal for both species.

15 Temporal disturbances are introduced by an additional mortality rate; they occur

16 after population growth and dispersal. Disturbances randomly reduce local density by

$17 D_{s}(t) \cdot N(t)$ individuals, where $D_{s}(t)$ ranges from 0 to $D_{s, \max }$ for each species and is

18 independently drawn in each time step.

\section{Simulation experiments and output}

21 We simulated population dynamics using reference values for the parameters if

22 not noted otherwise (cf. Tab. 1). Reproduction rates were set to 5 per time step, which 
1 seems reasonable for many species with low to medium body mass, e.g. for insects and

2 small mammals (Sinclair 1989). Local species' carrying capacity was 320 individuals.

3 In the time series analyses of species' densities (Figs. 1-3), we defined long-term

4 growth trends by estimating locally weighted linear fits for population sizes as a

5 function of time using a loess smoother (function loess in R 2.2.0, R Development Core

6 Team 2005). We measured the amplitudes of the over-compensating species' density

7 fluctuations via the absolute differences in the densities between consecutive time steps.

8 Again using a loess smoother for a locally weighted fit, we then analyzed how the

9 growth trends depend on amplitudes.

10 To investigate the role of niche overlap we varied the competition coefficient $a$

11 from 0 (no niche overlap) across 1 (complete overlap) to 1.2 (interspecific competition

12 stronger than intraspecific competition, cf. Fig. 4).

13 For the further and more extensive simulation experiments on the influence of

14 the growth model and space on coexistence (cf. Figs. 5, 6), we varied density regulation

15 from under-compensatory (minimum: $\ln (b)=-2$ ) to strong over-compensatory density

16 regulation (maximum: $\ln (b)=2$ ) for both species. For each combination of density

17 regulation types, 100 simulations each with 1000 time steps were conducted, and from

18 these we derived the survival probability of each species as well as the coexistence

19 probability (proportion of simulations where both species survived 1000 time steps). To

20 validate coexistence probabilities we additionally analyzed invasibility and monitored

21 the probability to invade the population of the respective competitor with very few

22 invaders. Both analyses gave comparable results, and thus we only report coexistence

23 probabilities. We further repeated the experiments under the exclusion of demographic

24 stochasticity. We found no qualitative changes in the results and thus report only those 
1 including stochasticity.

2 The simulation models were implemented with the Borland $\mathrm{C}++$ Builder 5 using

3 numerical routines from the GNU Scientific Library (GSL Team 1992) for the

4 probability distributions. Graphics and statistics were generated in $\mathrm{R} 2.2 .0$ (R

5 Development Core Team 2005). 


\section{$1 \quad$ 3. Results}

2 The simulations regarding coexistence of two species with equal competition coef-

3 ficients $(a=1$, i.e. conspecific individuals cause the same competitive pressure as

4 heterospecific individuals) showed that competitive interactions of two identical

5 compensators $(\ln (b)=0)$ resulted in the random extinction of one species (Fig. 1a). The

6 same was true for two identical over-compensators. However, when a compensating

$7 \quad(\ln (b)=0)$ and an over-compensating species $(\ln (b)=1.5)$ competed with each other,

8 both species were able to coexist (Fig. 1b, c).

9 Interspecific interactions dampened the fluctuations of the over-compensator, i.e.

10 amplitudes were much larger without interspecific interactions $(a=0$, Fig. 2a) than with

11 interactions $(a=1$, Fig. 1b, c). More specifically, amplitudes were buffered most

12 strongly when the compensating species was more frequent than the over-compensating

13 species. In contrast, amplitudes of the compensator were comparably strong without

$14(a=0$, Fig. 2b) and with interspecific interactions $(a=1$, Fig. 1). The local regression

15 analysis between the over-compensator's amplitudes and species' growth trends

16 revealed two alternating and mutually advantageous dynamic situations for the species

17 (Fig. 3): (1) low to moderate amplitudes of the fluctuations resulted on average in a

18 positive growth trend of the over-compensator but in a negative growth trend of the

19 compensator; (2) for strong amplitudes, the situation was vice versa. This was found to

20 be a general pattern, but the absolute magnitude of the amplitude where the switch from

21 positive to negative growth rates for the over-compensator and the competitor occurred

22 (i.e., where both species displayed zero growth) varied between simulations. However,

23 within each simulation the switch point of zero growth was identical for both the over-

24 compensator and the competitor. 
1 In addition to the experiments where intraspecific was equal to interspecific 2 competition strength $(a=1)$ and those without interspecific interactions $(a=0)$, we 3 gradually increased the competition coefficient $a$ (Fig. 4). Species with equal density 4 regulation types (i.e., equal $b$ ) were able to coexist only if interspecific competition was 5 weaker than intraspecific competition ( $a<1$, Fig. 4a, left side of vertical line). How 6 weak it had to be depended on the type of density regulation. However, if species 7 differed in density regulation types and one species showed over-compensation, 8 coexistence was possible even if interspecific was equal to or stronger than intraspecific 9 competition $(1<a<1.2$, Fig. $4 b$, right side of vertical line). In particular, interspecific 10 competition allowed a strong over-compensator that could not persist in isolation to 11 coexist with another species (Fig. 4b, extinction of a strong over-compensator at $12 a<0.35$, but survival and coexistence with an under-compensator at $0.35<a<1.2$ ).

13 Hence, interspecific competition clearly broadened the survival range that resulted from 14 the various density regulation types.

15 In a next step, we conducted a comparative analysis for a range of demographic 16 parameters and growth models (Fig. 5) and analysed for which combinations of density 17 regulation types coexistence was possible. This consistently occurred when at least one 18 species showed over-compensation and the other species differed in its density 19 regulation type (Fig. 5, green area). No coexistence occurred when both species had the 20 same density regulation type (cf. cells along the diagonals). From under-compensation 21 to moderate over-compensation, the species with stronger over-compensation was 22 superior, i.e. species A outcompeted species B (Fig. 5, yellow areas), but from moderate 23 to strong over-compensation the species with less over-compensation was superior, i.e. 24 species B outcompeted species A (Fig. 5, blue areas). Increasing the carrying capacity 
1 generally increased the number of density regulation types that were able to coexist with

2 each other. For small carrying capacities coexistence was either reduced to very small

3 ranges or was not possible at al (cf. plots for $K=200$ vs. $K=1000$ ).

4 The magnitude of the influence of growth rate depended on the growth model

5 that was chosen. While for the Ricker model coexistence was possible at small

6 reproduction rates (cf. plots with $R=2$ ) and decreased at high reproduction rates

$7 \quad(R=20)$, it was vice versa for the Hassell and the Gompertz type models. The MSS

8 model output was very robust to the choice of reproduction rate. Generally, at low

9 reproduction rates coexistence shifted to higher $b$ values. Too low carrying capacities

10 did not allow for coexistence in any model.

11 In a final comparison, we evaluated the role of space for coexistence (cf. Fig. 6).

12 We used growth rates that led to robust coexistence in homogeneous space $(R=2$ for

13 the Ricker model and $R=20$ for all other models). Global carrying capacity was set to

14500 (resulting in local carrying capacities of 125 individuals in the four patches).

15 Generally, fragmenting the available carrying capacity led to decreased coexistence.

16 However, coexistence was still much more probable than could be expected in a

17 completely isolated patch with a carrying capacity of 125 individuals. We further found

18 that increasing emigration rates resulted in decreased coexistence range (cf. plots with

$19 P_{e m i}=0.05$ vs. $\left.P_{e m i}=0.4\right)$. Including dispersal mortality slightly increased the

20 coexistence range through over-compensation. Additionally, a new coexistence

21 mechanism emerged: equal or very similar species (along the negative diagonal) were

22 able to 'neutrally' coexist, a mechanism well known from the literature (cf. Chesson

23 1994; Chesson 2000; Adler 2007). Qualitatively, results did not vary between different

24 growth models with the exception that coexistence was more robust to high emigration 
1 rates for the MSS and Gompertz type models than for the Ricker and Hassell models. 


\section{4. Discussion}

2

3 (identical in their growth rates, carrying capacities and competition coefficients) are

4 able to coexist if (1) they differ in their types of density regulation and (2) at least one

5 species shows over-compensating density regulation. We found that this coexistence

6 occurs over a wide parameter range of density regulation types and proved robust to

7 model choice, temporal disturbances and spatial structure.

\subsection{Nonlinear population growth maintains coexistence}

Our analysis reveals the underlying dynamic mechanism (Fig. 3): The resulting community dynamics are characterised by two dynamically alternating and mutually advantageous biotic system states. These states are generated internally, and the different fluctuation strength of the over-compensator's population density in these states is the key element. At low to moderate fluctuations, the over-compensator is the stronger competitor with a higher effective growth trend compared to the competitor (Johansson and Sumpter 2003). This leads to increasing dominance of the overcompensator's density in the population. However, high densities amplify the overcompensator's intrinsic fluctuations. At strong fluctuations, the competing species can use the emerging density depressions as temporal niches, provided that its density regulation is more compensatory. Consequently, the competing species has the higher effective net growth rate and increases its relative frequency in the population. However, the over-compensator's fluctuations are buffered at a high frequency of the competing species, and thus the over-compensator becomes the stronger competitor again. In sum, coexistence through over-compensation is possible because the over- 
1 compensator tends to generate fluctuations from which it suffers and the competing

2 species tends to dampen these fluctuations from which it benefits (Figs. 1, 3). These

3 alternating system stages can maintain coexistence both for competition coefficients

4 (i.e. degrees of niche overlap) that would lead to competitive exclusion in the classical

5 Lotka-Volterra equations (with linear density dependence; Fig. 4) and for strong over-

6 compensators that could not survive on their own due to strong endogenous fluctuations

7 and resulting deterministic extinction in the absence of a buffer (Fig. 4b).

8 Our simulation experiments show that various models that are well-established

9 in theoretical population ecology (e.g., Maynard Smith and Slatkin, Hassell, Gompertz,

10 Ricker) can display fluctuating coexistence through over-compensatory dynamics

11 similar to the oscillating coexistence in the more complex consumer-resource models

12 where the dynamics of consumers and resources are coupled explicitly (e.g., Armstrong

13 and McGehee 1980, Huisman and Weissing 2001, Abrams and Holt 2002, Wilson and

14 Abrams 2005). As coexistence through over-compensation results from differences in

15 the shape parameter $b$ of the growth function, it can be associated easily with Chesson's

16 category of relative nonlinearity (Chesson 1994; Chesson 2000). Also, Kuang and

17 Chesson (2008) suggested a similar interplay between two alternating states for a plant

18 community facing seed predation. In their model the relative nonlinearity of the growth

19 functions was generated by life history trade-offs between seed productivity and

20 persistence in the seed bank rather than through density regulation. These different

21 studies highlight that considering not only the size of competition coefficients but also

22 the nonlinearity of population growth promises new insights into coexistence dynamics.

$24 \quad 4.2$ Robustness of coexistence 
1 Our sensitivity analysis demonstrates that coexistence through over-compensation is

2 a feature of all four growth models, albeit for slightly different parameter ranges. As

3 endogenous density fluctuations result from the combined action of population growth

4 rate $R$, density regulation type $b$ and the particular functional form of the population

5 growth model, different models lead to different coexistence ranges (Fig. 5). This

6 explains for example why the Ricker model exhibits coexistence at smaller $R$ than the

7 Hassel model, or why the Gompertz model needs larger $R$ than the MSS model to

8 maintain coexistence. Furthermore, our results show that only relatively large carrying

9 capacities can support coexistence through over-compensation. This is because large

10 mean population sizes buffer density fluctuations and help to prevent extinction during

11 density drops. Thus, our results suggest that communities relying on this coexistence

12 mechanism are likely to be sensitive to landscape fragmentation, in particular if

13 remaining patches are small. Yet, it is not high connectivity between patches (i.e., when

14 species have high emigration rates and low dispersal mortalities) that buffers the

15 fragmentation effect best, but low connectivity (low emigration rates, Fig. 6). The

16 reason is that coexistence through over-compensation depends on interacting and

17 alternating dynamics of the two species (as explained above). Too much exchange of

18 individuals between patches disturbs these dynamics and reduces coexistence. Thus,

19 limited dispersal is advantageous because it leaves local dynamics almost undisturbed

20 while still allowing for recolonization of extinct small patches, which supports

21 coexistence. This is quite different from coexistence through limited-dispersal

22 specialization where individuals disperse locally, either to exploit empty patches

23 quickly or to more rapidly increase densities in neighbouring patches to exclude

24 competitors (e.g. Bolker and Pacala 1999). Coexistence through over-compensation in 
1 fragmented landscapes is a good example for a situation where a weak stabilizing

2 mechanism (coexistence through over-compensation cannot develop its full power due

3 to the small patch sizes) is supported by limited dispersal and only the two mechanisms

4 together lead to long-term coexistence (Chesson 2000; Adler 2007).

\subsection{Relevance for real ecosystems}

7 What is the relevance of this coexistence mechanism for real ecosystems? The type

8 of density regulation is a species trait that is difficult to measure in field experiments

9 (Godfray et al. 1990; Morris 1990). Even for time series derived from simulation

10 models, it is difficult to estimate the type of density regulation without an a priori

11 knowledge of the processes included in the model. In spite of these difficulties, many

12 researchers agree that different types of density regulation may have a significant effect

13 on ecosystem dynamics and functioning (reviewed in Hastings et al. 1993). For

14 example, several studies have provided evidence of chaotic dynamics due to over-

15 compensatory density regulation, in experimental as well as 'natural' conditions

16 (Godfray et al. 1990; Hastings et al. 1993). Field studies suggest over-compensating

17 density regulation as a possible mechanism in the competition for resources especially

18 for species with density regulation in early life stages (Sinclair 1989; e.g. for butterflies

19 such as cinnabar moths Tyria jacobaeae, beetles such as southern pine beetle

20 Dendroctonus frontalis, Reeve et al. 1998, or small mammals such as Arvicola

21 terrestris, Aars et al. 2001, and Microtus ochrogaster, Getz et al. 2006). We suggest

22 that particularly in communities with high species diversity but no apparent

23 differentiation in physiological characteristics or resource requirements, species

24 coexistence may rely critically on the differentiation in the types of density regulation as 
1 demonstrated in our theoretical investigations. Examples may be the high small

2 mammal and insect biodiversity in some parts of the tropical rainforest zone or marine

3 plankton communities

4

5 In conclusion, we were able to show that the essence of coexistence through

6 over-compensation is the alternation of different dynamic community states emerging

7 from interacting species of different density regulation types. Coexistence is maintained

8 for a wide range of combinations of density regulation types, and it is also robust with

9 respect to the introduction of temporal disturbances and spatial structure as well as to

10 the choice of the particular non-linear model. Nevertheless, interesting questions for

11 future research remain, e.g., how these mechanisms may work if more species and

12 resources were involved, or how it could evolve in communities.

13 Our results clearly suggest that theoretical community ecology should go beyond

14 the linear density dependence of Lotka-Volterra equations. For example, for a better

15 understanding of spatial coexistence mechanisms it may be helpful to consider the

16 relevance of nonlinear density dependence for both local processes and resulting

17 equations for mean densities (moment equations, cf. Bolker and Pacala 1999).

18 Moreover, field studies should examine more closely competition types within

19 communities when studying coexistence. As scramble competition may lead to over-

20 compensating density regulation, a more detailed analysis of scramble versus contest

21 competition may give further insights into the functioning of communities.

23 Acknowledgements:

24 We thank T. Mueller, J. Groeneveld and B. Reineking for stimulating discussions and 
1 useful comments on earlier versions of this manuscript. We thank Jim Grover for very

2 helpful comments on an earlier version and the title of the manuscript. Suggestions of

3 anonymous reviewers helped to clarify and improve our manuscript considerably. We

4 appreciate the financial support provided by the BMBF Germany (German Federal

5 Ministry of Education and Research, project ID: 01 LB 0202). 
1

2

3 Aars, J., Lambin, X., Denny, R. and Griffin, A. C., 2001. Water vole in the Scottish

\section{References} uplands: distribution patterns of disturbed and pristine populations ahead and behind the American mink invasion front. Anim. Conserv. 4, 187-194.

Abrams, P. A. 2004. When does periodic variation in resource growth allow robust coexistence of competing consumer species? Ecology 85 (2), 372-382.

Adler, F. R. 1990. Coexistence of 2 types on a single resource in discrete-time. J. Math. Biol. 28 (6), 695-713.

Adler, P.B., HilleRisLambers, J., Levine, J.M. 2007. A niche for neutrality. Ecol. Lett. $10,95-104$.

Anderies, J. M. and Beisner, B. E., 2000. Fluctuating environments and phytoplankton community structure: A stochastic model. Am. Nat. 155 (4), 556-569.

Armstrong, R. and McGehee, R., 1976. Coexistence of two competitors on one resource. J. Theor. Biol. 56, 449-502.

Armstrong, R. and McGehee, R., 1980. Competitive exclusion. Am. Nat. 115, 151-170.

Bolker, B. M. and Pacala, S. W. 1999. Spatial moment equations for plant competition: understanding spatial strategies and the advantages of short dispersal. Am. Nat. 153 (6), 575-602.

Chesson, P. 2000. Mechanisms of maintenance of species diversity. Annu. Rev. Ecol. Syst. 31, 343-366. 
1 Chesson, P. 1994. Multispecies competition in variable environments. Theor. Popul.

$2 \quad$ Biol. $45(3), 227-276$.

3 Chesson, P. 2003. Understanding the role of environmental variation in population and

4 community dynamics - Introduction. Theor. Popul. Biol. 64 (3), 253-254.

5 Cushing, J. M., Henson, S. M. and Blackburn, C. C., 2007. Multiple mixed-type

6 attractors in a competition model. Journal of Biological Dynamics 1 (4), 347-362.

7 Damgaard, C. 2004. Dynamics in a discrete two-species competition model: coexistence 8 and over-compensation. J. Theor. Biol. 227 (2), 197-203.

9 Edmunds, J., Cushing, J. M., Costantino, R. F., Henson, S. M., Dennis, B. and 10 Desharnais, R. A., 2003. Park's Tribolium competition experiments: a non11 equilibrium species coexistence hypothesis. J. Anim. Ecol. 72 (5), 703-712.

12 Gause, G. F. 1934. The struggle for existence, The Williams \& Wilkins Co., Baltimore.

13 Getz, L. L., Oli, M. K., Hofmann, J. E. and McGuire, B., 2006. Vole population 14 dynamics: Factors affecting peak densities and amplitudes of annual Microtus 15 ochrogaster population fluctuations. Basic and Applied Ecology 7 (2), 97-107.

16 Getz, W. M. 1996. A hypothesis regarding the abruptness of density dependence and the 17 growth rate of populations. Ecology 77 (7), 2014-2026.

18 Godfray, H. C. J., Blythe, S. P., Williamson, M. and Perry, J. N., 1990. Complex 19 dynamics in multispecies communities. Phil. Trans. R. Soc. Lond. B 330 (1257), $20 \quad 221-233$. 
1 Grover, J. P. 1990. Resource competition in a variable environment - phytoplankton

2 growing according to monods model. Am. Nat. 136 (6), 771-789.

3 GSL $\quad$ Team, $1992 . \quad$ GNU Scientific Library.

4 http://www.gnu.org/software/gsl/manual/html_node/.

5 Hanski, I. and Woiwod, I. P., 1993. Spatial synchrony in the dynamics of moth and 6 aphid populations. J. Anim. Ecol. 62, 656-668.

7 Hassell, M. P. and Comins, H. N., 1976. Discrete-time models for 2-species 8 competition. Theor. Popul. Biol. 9 (2), 202-221.

9 Hastings, A., Hom, C. L., Ellner, S., Turchin, P. and Godfray, H. C. J., 1993. Chaos in 10 ecology: Is mother nature a strange attractor? Annu. Rev. Ecol. Syst. 24, 1-33.

11 Huisman, J., Johansson, A. M., Folmer, E. O. and Weissing, F. J., 2001. Towards a 12 solution of the plankton paradox: the importance of physiology and life history. $13 \quad$ Ecol. Lett. $4(5), 408-411$.

14 Huisman, J. and Weissing, F. J., 1999. Biodiversity of plankton by species oscillations 15 and chaos. Nature 402 (6760), 407-410.

16 Hutchinson, G. E. 1961. The paradox of the plankton. Am. Nat. 95 (882), 137-145.

17 Johansson, A. and Sumpter, D. J. T., 2003. From local interactions to population 18 dynamics in site-based models of ecology. Theor. Popul. Biol. 64 (4), 497-517. 
1 Johst, K., Berryman, A. and Lima, M., 2008. From individual interactions to population

2 dynamics: individual resource partitioning simulation exposes the causes of

3 nonlinear intra-specific competition. Population Ecology 50, 79-90.

4 Kaitala, V., Ylikarjula, J. and Heino, M., 1999. Dynamic complexities in host-parasitoid

$5 \quad$ interaction. J. Theor. Biol. 197 (3), 331-341.

6 Kuang, J. J. and Chesson, P., 2008. Predation-competition interactions for seasonally 7 recruiting species. Am. Nat. 171, E119-E133.

8 Lotka, A. J. 1925. Elements of physical biology, Williams and Wilkins, Baltimore.

9 Lundberg, P., Ranta, E., Ripa, J. and Kaitala, V., 2000. Population variability in space 10 and time. Trends Ecol. Evol. 15 (11), 460-464.

11 May, R. M. 1975. Biological populations obeying difference equations: stable points, 12 stable cycles, and chaos. J. Theor. Biol. 51, 511-524.

13 May, R. M. 1976. Simple mathematical models with very complicated dynamics. $14 \quad$ Nature 261, 459-467.

15 Maynard Smith, J. and Slatkin, M., 1973. The stability of predator-prey systems. $16 \quad$ Ecology 54, 384-391.

17 Morris, W. F. 1990. Problems in detecting chaotic behavior in natural populations by 18 fitting simple discrete models. Ecology 71 (5), 1849-1862. 
1 Münkemüller, T. and Johst, K., 2006. Compensatory versus over-compensatory density

2 regulation: implications for metapopulation persistence in dynamic landscapes.

3 Ecol. Model. 197 (1-2), 171-178.

4 Münkemüller, T. and Johst, K., 2007. How does intraspecific density regulation

5 influence metapopulation synchrony and persistence? J. Theor. Biol. 245 (3), 553-

6563.

7 Murrell, D. J., Travis, J. M. J. and Dytham, C., 2002. The evolution of dispersal 8 distance in spatially-structured populations. Oikos 97 (2), 229-236.

9 Neuhauser, C. P. and Pacala, S. W., 1999. An explicitly spatial version of the Lotka-

10 Volterra model with interspecific competition. Ann. Appl. Probab. 9 (4), 1226-1259.

11 R Development Core Team, 2005. R: a language and environment for statistical 12 computing. http://www.R-project.org.

13 Reeve, J. D., Rhodes, D. J. and Turchin, P., 1998. Scramble competition in the Southern 14 pine beetle, Dendroctonus frontalis. Ecol. Entomol. 23 (4), 433-443.

15 Ripa, J. and Lundberg, P., 2000. The route to extinction in variable environments. Oikos $90(1), 89-96$.

17 Schippers, P., Verschoor, A. M., Vos, M. and Mooij, W. M., 2001. Does 'supersaturated coexistence' resolve the 'paradox of the plankton'? Ecol. Lett. 4 (5), 404-407.

19 Shmida, A. and Ellner, S., 1984. Coexistence of plants with similar niches. Vegetatio $58,29-55$. 
1 Sinclair, A. R. E. 1989. Population regulation in animals, in: Cherrett, J. M., Bradshaw,

2 A. D., Goldsmith, F. B., Grubb, P. J. and Krebs, J. R. (Eds.), Ecological concepts:

3 the contribution of ecology to an understanding of the natural world. Blackwell

4 Scientific Pub., Oxford, London, Edinburgh, pp. 197-242.

5 Smith, H. L. 1981. Competitive coexistence in an oscillating chemostat. Siam Journal 6 on Applied Mathematics 40 (3), 498-522.

7 Szabo, P. and Meszena, G., 2006. Limiting similarity revisited. Oikos 112 (3), 612-619.

8 Volterra, V. 1926. Fluctuations in the abundance of a species considered 9 mathematically. Nature $188,558-560$.

10 Zicarelli, S. 1975. Mathematical analysis of a simulation model with several predators 11 on a single prey, University of Minnesota,

12

13 
1 Table 1: Overview of parameters that were varied across simulation experiments,

2 parameters that were kept constant across all simulation experiments (but some were 3 drawn from given distributions), and output variables.

4 
1 Figure 1: Population densities of two competing species over 300 time steps on one

2 patch (for higher detail only the first 300 of 1000 time steps are shown): (a) equal types

3 of density regulation without disturbances, (b) different types of density regulation

4 without disturbances and (c) different types of density regulation with disturbances.

5 Intraspecific competition is as strong as interspecific competition $(a=1)$.

7 Figure 2: Population densities of two non-interacting species over 300 time steps on one

8 patch (for better detail only the first 300 of 1000 time steps are shown): (a)

9 compensating density regulation and (b) over-compensating density regulation.

11 Figure 3: Long-term trends in population growth of both species depend on the over12 compensator's fluctuation amplitudes. The figure provides an example for competing 13 species on one patch with disturbances (cf. Fig. 1c, Fig. 4b). The vertical line marks the 14 switch at which the growth trends of both species change their algebraic sign.

16 Figure 4: Coexistence probability of two species over 1000 time steps for different 17 competition coefficients and different density regulation types (uc: under-compensation, $18 \ln (b)=-1$, c: compensation; $\ln (b)=0$, moc: moderate over-compensation, $\ln (b)=1.5$, and 19 soc: strong over-compensation, $\ln (b)=2$ ). In (a) the competing species have equal types 20 of density regulation and in (b) types of density regulation differ. The figures provide an 21 example for competing species on one patch with disturbances (cf. Fig. 1c, Fig. 3b). The vertical lines mark the value of $a$ where intraspecific equals interspecific competition 23 strength. 
1 Figure 5: Coexistence of two species (green) in a homogeneous landscape. Species

2 differ only in their types of density regulation $(\ln (b)<0$ : under-compensation, $\ln (b)=0$ :

3 compensation, $\ln (b)>0$ : over-compensation). We compared different population growth

4 models (in the columns: Maynard Smith and Slatkin, Hassell, Ricker, Gompertz type) at

5 different values for reproduction rates $(R=2$ or 20$)$ and carrying capacities $(K=200,500$

6 or 1000). The colours code the competition outcome: green marks coexistence

7 probability $\geq 0.2$, yellow marks coexistence probability $<0.2$ and persistence

8 probability of species $\mathrm{A} \geq 0.5$, blue marks coexistence probability $<0.2$ and persistence

9 probability of species $\mathrm{B} \geq 0.5$, and grey marks simulations where none of the conditions

10 is fulfilled. The presented output for $b_{A} \geq b_{B}$ is equal to the output of $b_{A} \leq b_{B}$ because

11 species only differ in $b$ (white marks not simulated parameter combinations).

13 Figure 6: Coexistence of two species (green) in a fragmented landscape with four 14 patches. Species differ only in their types of density regulation $(\ln (b)<0$ : under15 compensation, $\ln (b)=0$ : compensation, $\ln (b)>0$ : over-compensation). We compared 16 different population growth models (in the columns: Maynard Smith and Slatkin, 17 Hassell, Ricker, Gompertz type) at different values for dispersal mortality ( $m=0$ or 0.2 )

18 and emigration rates $\left(P_{\text {emi }}=0.05,0.1\right.$ or 0.4$)$. The colours code is the same as in Fig. 5. 


\begin{tabular}{|c|c|c|c|}
\hline \multicolumn{4}{|c|}{ Parameters that varied across simulation experiments } \\
\hline Symbol & Parameter & Reference & Experiments \\
\hline$R$ & Reproduction rate & 5 & {$[2 ; 20]$} \\
\hline$a$ & Competition coefficient & 1 & {$[0 ; 1.2]$} \\
\hline & Local carrying capacities & 320 & {$[125 ; 1000]$} \\
\hline$b_{A}, b_{B}$ & Type of density dependence & {$\left[\mathrm{e}^{-2} ; \mathrm{e}^{2.4}\right]$} & {$\left[\mathrm{e}^{-2} ; \mathrm{e}^{2}\right]$} \\
\hline$m$ & Dispersal mortality rate & & 0 or 0.2 \\
\hline$p_{e m i}$ & Emigration rate & - & {$[0 ; 0.4]$} \\
\hline$P_{\text {Number }}$ & Patch number & 1 & 1 or 4 \\
\hline$D_{s, \max }$ & Max. disturbance mortality & 0.05 & 0 or 0.05 \\
\hline \multicolumn{4}{|c|}{ Parameters that were kept constant across all simulation experiments } \\
\hline \multirow[t]{2}{*}{ Symbol } & Parameter & \multicolumn{2}{|l|}{ Values } \\
\hline & Initial population size per species & \\
\hline$L_{\text {Size }}$ & Lattice size $\left[\mathrm{cells}^{2}\right]$ & \multicolumn{2}{|l|}{$20 * 20$} \\
\hline$D D$ & Mean dispersal distance [cells] & \multicolumn{2}{|l|}{$1,2,6,12,20$} \\
\hline \multicolumn{4}{|c|}{ Output variables } \\
\hline \multirow{4}{*}{$\frac{\text { Symbol }}{N(t)}$} & Variable & & \\
\hline & Individual number over time & & \\
\hline & Survival probability & & \\
\hline & Coexistence probability & & \\
\hline
\end{tabular}



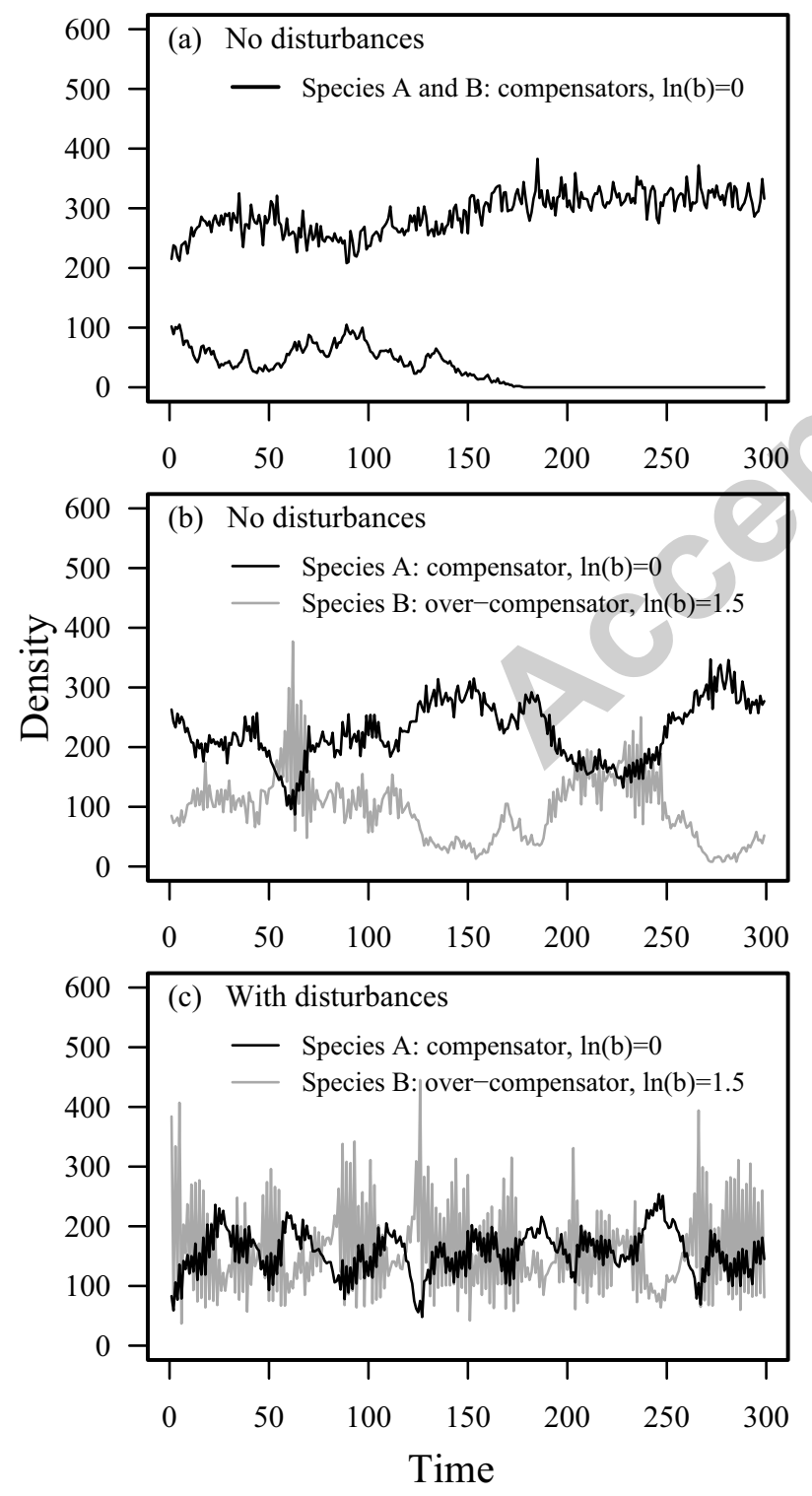


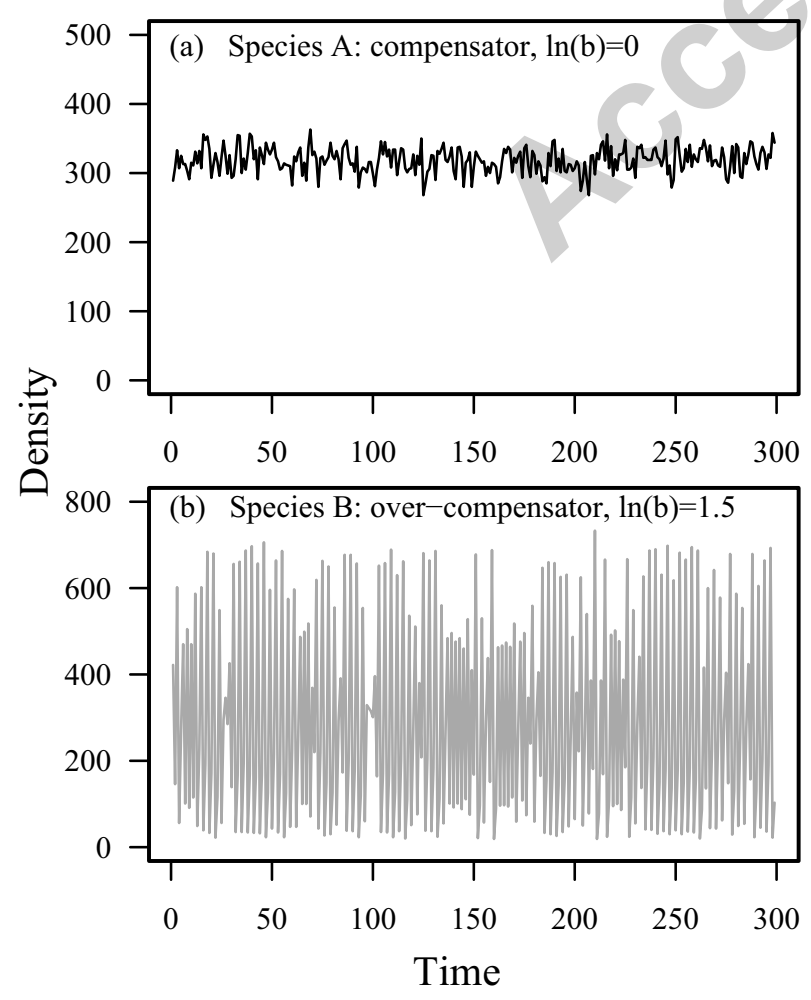


4. Figure

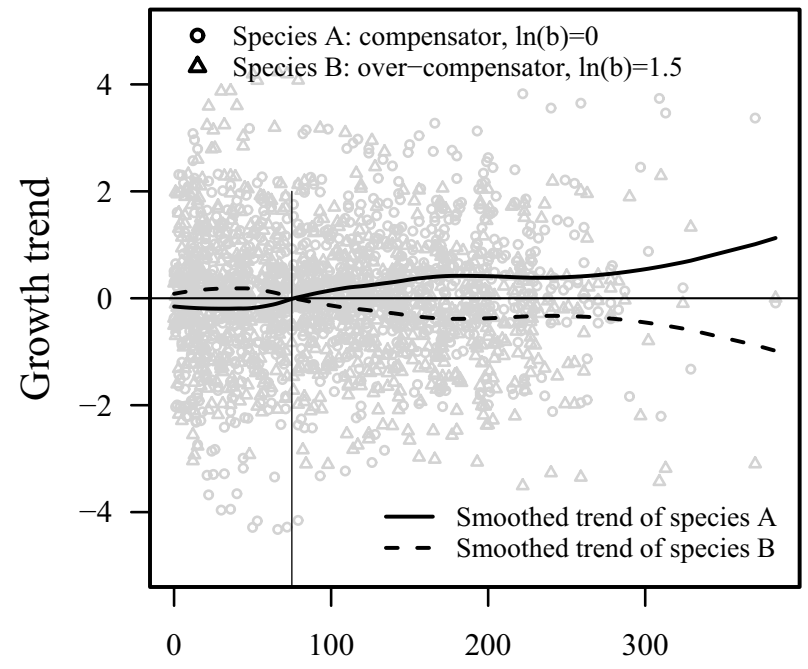

Fluctuation amplitude 
MSS
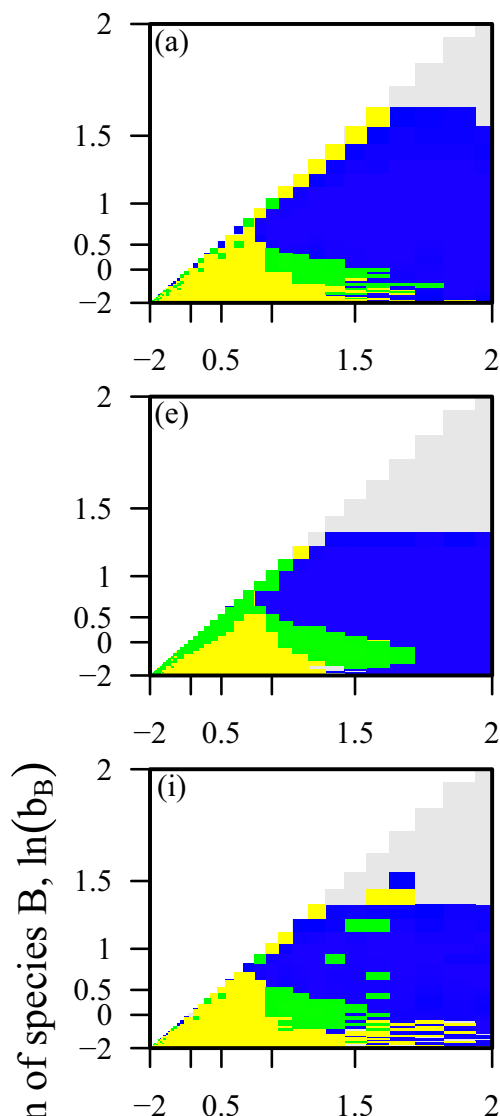

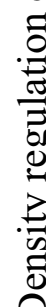

Hassell

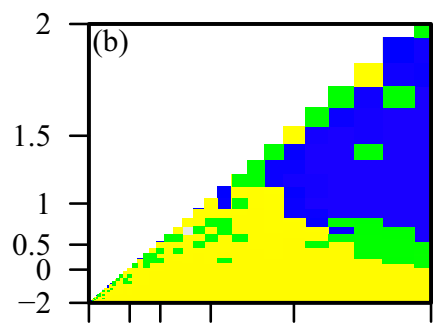

$\begin{array}{lll}2 & -2 & 0.5\end{array}$

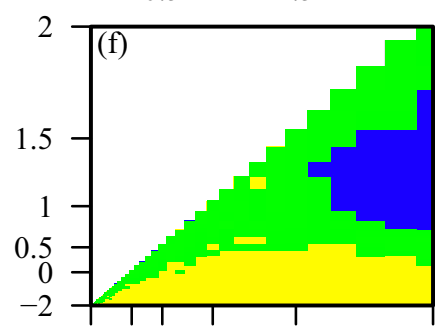

$\begin{array}{llll}2 & -2 & 0.5\end{array}$
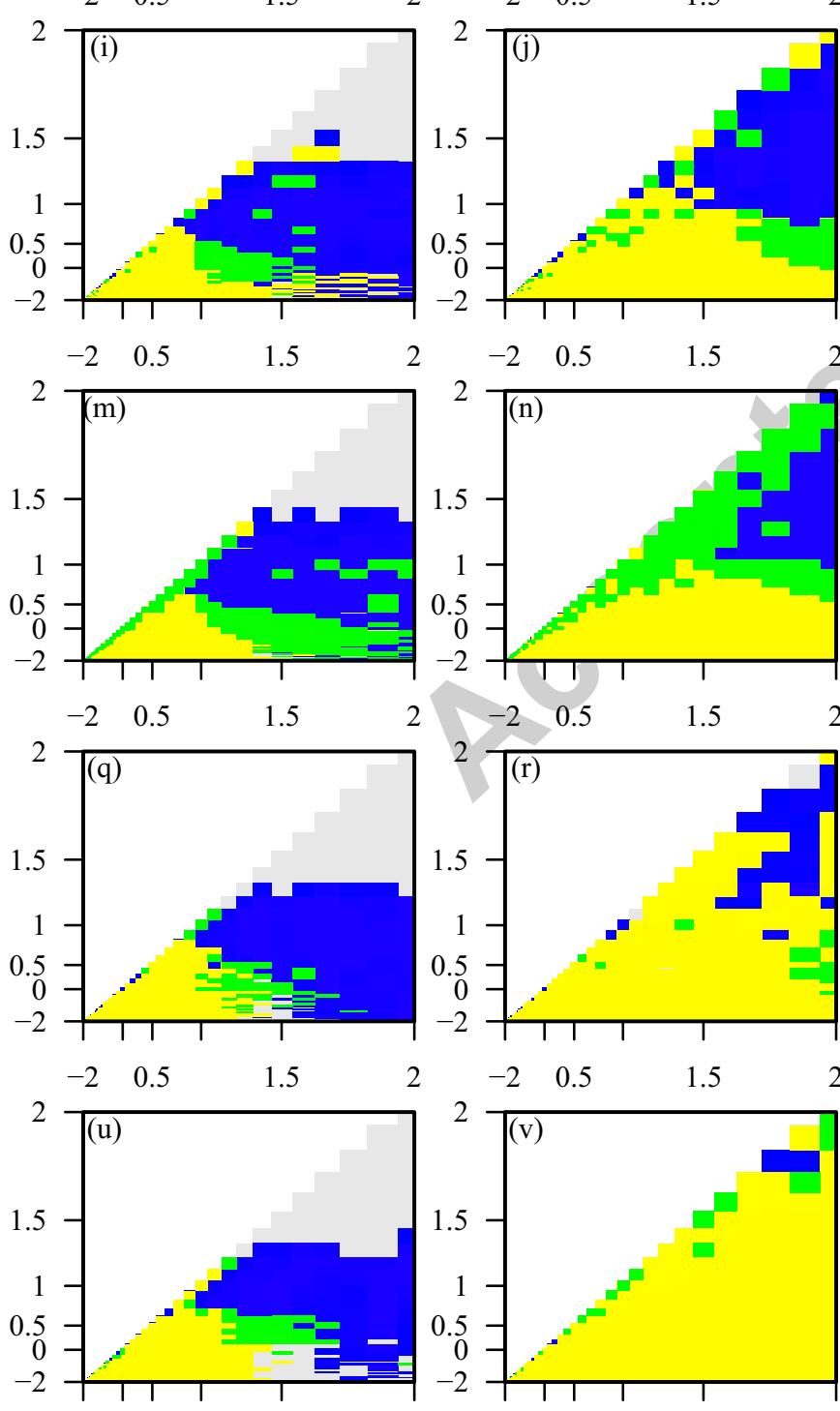

$\begin{array}{ll}-2 & 0.5\end{array}$
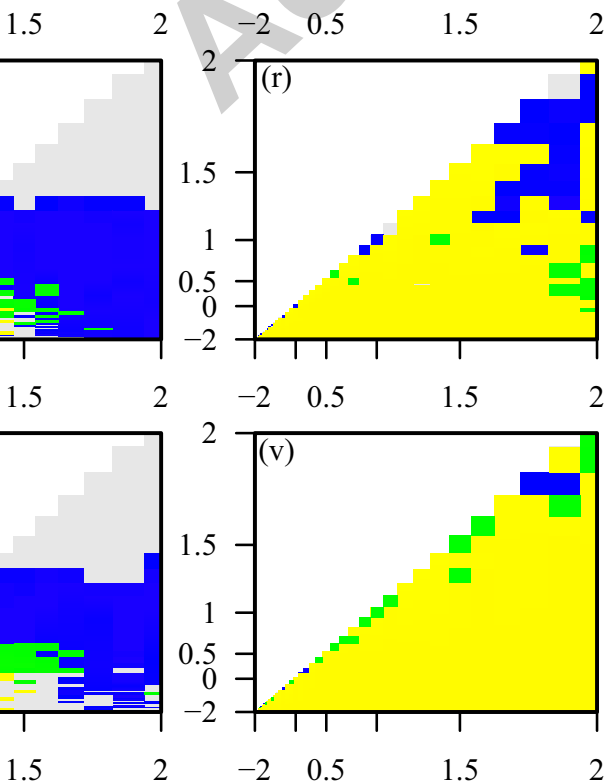

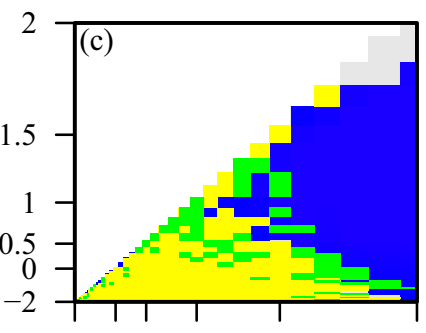

Ricker

Gompertz
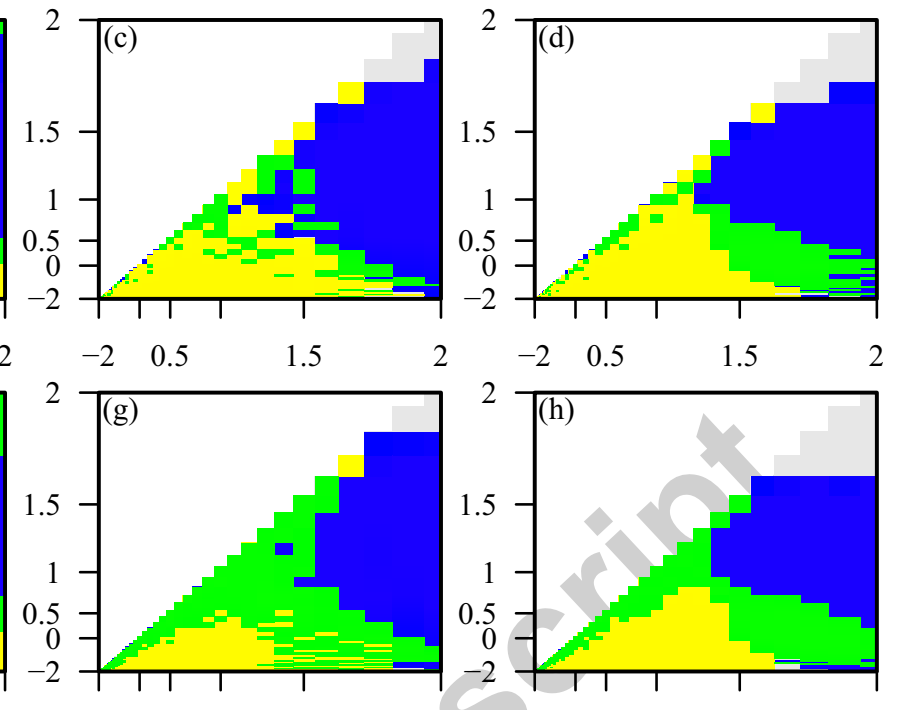

$\stackrel{0}{\sharp}$

$n$
0
0
0
0

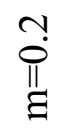
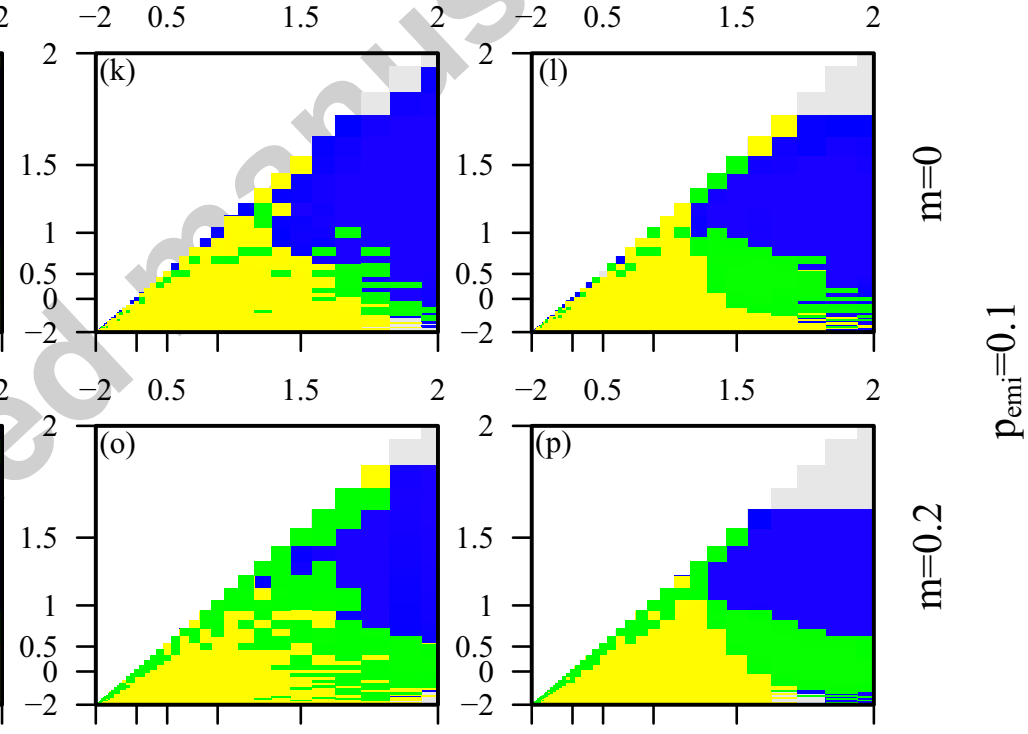

$\stackrel{N}{!}$
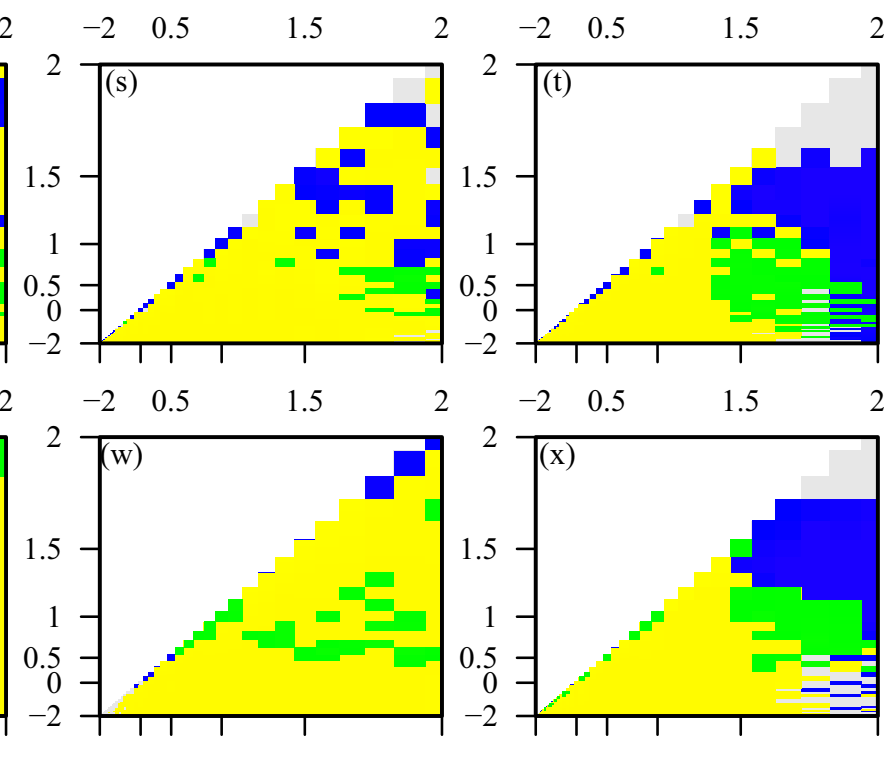

$\prod_{\Xi}$

$\stackrel{+}{0}$

$\stackrel{\text { ำ }}{\dddot{I}}$

Density regulation of species $A, \ln \left(b_{A}\right)$ 